\title{
Tolerability and performance of BIP endotracheal tubes with noble metal alloy coating - a randomized clinical evaluation study
}

\author{
Gunilla Björling ${ }^{1,2^{*}}$, Dorota Johansson ${ }^{3,4}$, Linda Bergström, ${ }^{3,4}$, Shah Jalal ${ }^{5}$, Ivar Kohn ${ }^{6}$, Claes Frostell ${ }^{2}$
} and Sigridur Kalman ${ }^{6,7}$

\begin{abstract}
Background: Hospital acquired infections worsen the outcome of patients treated in intensive care units and are costly. Coatings with silver or metal alloys may reduce or alter the formation of biofilm on invasive medical devices. An endotracheal tube (ETT) is used to connect the patient to a ventilator and coated tubes have been tested in relation to bacterial colonization and respiratory infection. In the present study, we aimed to evaluate and compare a coated and uncoated ETT for patient symptoms and local tracheal tolerability during short term clinical use. Degree of bacterial colonization was also described.

Methods: A silver-palladium-gold alloy coating ('Bactiguard ${ }^{\oplus \prime}$ Infection Protection, BIP) has been extensively used on urinary tract catheters and lately also on central venous catheters. We performed a randomised, single-blinded, controlled, first in man, post Conformité Européenne (EC) certification and CE marking study, focused on Bactiguard ${ }^{\circledR}$ coated ETTs (BIP ETT). Thirty patients at a tertiary university hospital scheduled for upper abdominal elective surgery with an expected duration of anaesthesia of at least $3 \mathrm{~h}$ were randomised; BIP ETT $(n=20)$ or standard ETT $(n=10)$. The tolerability was assessed with a modified version of Quality of Life Head and Neck Module, QLQ-H\&N35 and by inspection of the tracheal mucosa with a fibre-optic bronchoscope before intubation and at extubation. Adverse Events (AE) and bacterial adherence were also studied. Statistical evaluations were carried out with the Fisher's Exact Test, the Clopper-Pearson method, as well as a Proportional Odds Model.

Results: Differences between groups were identified in 2 of 8 patient related symptoms with regard to tolerability by QLQ-H\&N35 (cough, $p=0.022$ and dry mouth, $p=0.014$ in the treatment group.). No mucosal damage was identified with bronchoscopy. A low level of bacterial colonization with normal flora, equal between groups, was seen after short-term of intubation (median $5 \mathrm{~h}$ ). No serious Adverse Events related to the use of an ETT were observed. The results should be treated with caution due to statistical confounders, a small study size and large inter-individual variability in bacterial adhesion.

(Continued on next page)
\end{abstract}

\footnotetext{
* Correspondence: gunilla.bjorling@ki.se

${ }^{1}$ The Swedish Red Cross University College, P.O. Box 55676SE-10215

Stockholm, Sweden

${ }^{2}$ Karolinska Institutet, Department of Clinical Sciences, Division of Anaesthesia

and Intensive Care, Danderyd Hospital, SE-18288 Stockholm, Sweden

Full list of author information is available at the end of the article
} 
(Continued from previous page)

Conclusions: The new device BIP ETT is well tolerated and has good clinical performance during short-term intubation. Studies with larger sample sizes and longer intubation periods ( $>24 \mathrm{~h})$ in the ICU-setting are needed and can now be planned in order to identify possible differences in clinical outcomes.

Trial registration: Registered in ClinicalTrials.gov, Registration number: NCT01682486, Date of Registration: August, 30, 2012

Keywords: Endotracheal tube, Colonization, bacterial, Complication, Clinical method, Anaesthesia, Airway, Noble metal alloy coating

\section{Background}

Hospital acquired infections (HAI) are a global problem and among the four major causes of death in the Western world. Generally, $10 \%$ of hospitalised patients in the Western world acquire HAI [1-4]. Medical devices are rapidly colonized with bacteria during the formation of biofilm, which in turn can lead to health care-related infections [5-8]. But as the usage of medical technical equipment is necessary within the health care system, it is a particularly important area to study and optimize $[9,10]$.

To reduce the biofilm formation on endotracheal tubes, coatings with pure silver or silver compounds have been evaluated. The efficacy and safety of endotracheal tubes (ETTs) coated with pure silver or silver compounds have been reported in three randomised controlled clinical [11-13]. No safety issues were reported. The studies reported significant reduction of biofilm formation $[12,13]$ and as well as a delay of VAP. The noble metal alloy coating has been extensively used on urinary catheters and central venous lines [14-16]. This is a first in man study on an endotracheal tube with the Bactiguard ${ }^{\circledR}$ noble metal coating (BIP ETT).

The primary objective was to determine the tolerability of the medical device BIP ETT compared to standard uncoated ETTs in a small selected population of patients undergoing elective surgery. The secondary objective was to assess the overall safety and performance related to the medical device. The hypothesis was that the coated tube (BIP ETT) would be similar to the standard endotracheal tube with regard to patient-experienced symptoms and that the tracheal mucosa would be similarly affected by contact with the BIP ETT as by contact with a standard endotracheal tub. The primary endpoint was patient-reported symptoms in the throat and mouth, as well as visual appearances of the upper tracheal mucosa the day after tracheal intubation.

Though bacterial colonization is of major interest when it comes to medical devices inserted in the human body, this study is focused on the tracheal safety and tolerability when exposed to the new coating. Bacterial colonization after a few hours of endotracheal tube usage is described here and can be considered as a baseline in future studies with coated endotracheal tubes used for longer clinical periods (days).

\section{Methods \\ Study design}

This was a single-centre, randomised, single-blinded, and controlled, first in man, post Conformity Europermit (EC) certification and CE marking study. Patients undergoing elective surgery of the upper gastrointestinal tract with an expected duration of at least $3 \mathrm{~h}$ were asked to participate. The study was conducted at a Karolinska University Hospital in Sweden and approved by the Ethical Review Board in Stockholm (Dnr 20121205-31, protocol number 1842-2026-CDOC). Written informed consent from each patient was obtained before entry into the study. All patients signed a written informed consent before participating in the study. The study is outlined in clinicaltrials.gov database with the identifier: NCT01682486.

\section{Investigational device BIP ETT}

BIP ETT is an endotracheal tube designed for insertion through the mouth or nose. The, in- and outside surfaces are coated with the Bactiguard ${ }^{\circ}$ coating (Sweden) which consists of a noble metal alloy of silver, palladium and gold, which reduces bacterial adhesion in-vitro. The latter phenomenon is a contributing factor to microbial colonisation and biofilm formation, which in turn increases the risk for VAP. The BIP ETT is made from medical grade polyvinyl chloride (PVC). It is a sterile single use $100 \%$ latex free, two-lumen tube (one lumen connected to a high volume low pressure cuff with a standard connector), has a Magill curve and rounded tip with a Murphy eye.

\section{Control device}

Standard uncoated ETTs made of PVC, normally used for intubation at the investigators site (Mallinckrodt ${ }^{\mathrm{mm}}$ ETT) with standard labelling, were used as reference devices. They were sterile single use $100 \%$ latex free, two-lumen tubes (one lumen connected to a high 
volume low pressure cuff with a standard connector), had a Magill curve and rounded tip with a Murphy eye.

\section{Study population}

Inclusion criteria were adults $\geq 18$ years of age, requiring endotracheal intubation $\geq 3 \mathrm{~h}$ needing an endotracheal tube sized 7 or $8 \mathrm{~mm}$. Exclusion criteria were cognitive dysfunction (i.e., inability to understand and fill out the study questionnaires), transmissible blood disease, multiresistant bacterial colonization, current or continuous treatment with immune modulating therapies, e.g., systemic (or inhalation) use of cortisone or non-steroid anti-inflammatory drugs (NSAID), on-going respiratory infection, as well as deficient poor skills in writing and or speaking Swedish.

\section{Screening and randomization}

The list of scheduled major surgery eligible patients were identified and scanned and thereafter screened by reading the patients' journal. Eligible patients were later approached for information and consent. In total, 30 patients were screened and 30 patients were enrolled in the study. The enrolled patients were randomised to airway management during anaesthesia and surgery with either the BIP ETT $(n=20)$ or standard ETT $(n=10)$. The randomisation was designed to distribute the patients in a ratio of 2:1 in favour of the BIP ETT group. One patient in the BIP ETT group was prematurely withdrawn from the study due to vomiting during induction of anaesthesia. The patient then needed a rapid sequence induction and therefore, pre-intubation bronchoscopy could not be carried out. Therefore, the patient was withdrawn from the study.

\section{Endpoints}

\section{Primary endpoints}

The difference between the treatments (BIP ETT versus standard ETT) for each of the 8 questions in the modified European Organization for Research and Treatment of Cancer (EORTC) Quality of Life Questionnaire for Head \& Neck with 35 questions (QLQ H\&N35) [18], on the day after tracheal intubation.

The difference between treatments (BIP ETT versus standard ETT) in photo assessment (blinded assessment), and direct assessment (unblinded assessment) of the surface tracheal lining, before intubation and after extubation.

\section{Secondary endpoints}

Safety assessed based on adverse events (AE) and serious AEs (SAEs) and on any problems occurring during the post-operative course (Yes/No).

Performance assessed by bacterial colonization of BIP ETT versus standard ETT, microbiological testing of bacteria from a defined area of the ETT surface ex vivo (quantification and typing).

\section{Data collection}

Demographics, smoking habits, medical history of significance, and concomitant medication were assessed prior to surgery. The study comprised of four visits; a telephone contact by a study nurse to inform the patient about the study and send the written patient information to the patient up to 28 days prior to screening (Visit 1); a screening visit 1 to 14 days prior to surgery when also the informed consent form was signed and demographic data, medical history gathered and physical examination was performed (Visit 2); the actual day of surgery/intubation (Visit 3), and a follow-up on the day after surgery (Visit 4).

The study intervention was carried out at Visit 3. As the patient arrived in the operating theatre, local routines were followed. No lidocaine or lubricating jellies were used in conjunction with the ETT. Prior to intubation of the ETT, the endotracheal cuff was inflated as a test. The intubation was carried out after a bronchoscopy where the ETT was pulled over the fiberscope, and the cuff pressure was kept below $20 \mathrm{cmH}_{2} \mathrm{O}$ if possible. Endotracheal cuff pressures were recorded using an intra-cuff monitor, and the pressure was recorded during the anaesthesia. After induction of anaesthesia, the number of intubation attempts and the need for other intubation devices were recorded, as well as any injuries to lip, tongue or teeth. The ETT was not to be repositioned unless necessary. During extubation, a bronchoscope was again placed in the trachea through the ETT. The ETT was then removed and the trachea inspected. The time for intubation and extubation, as well as the type of fibre-optic bronchoscope were noted. The medical techniques and aseptic procedures used were according to currently accepted practice.

\section{Assessments of tolerability}

To assess the tolerability of the BIP ETT, a modified version of the head and neck module of the questionnaire developed for assessing quality of life was used (Quality of Life Head and Neck Module, QLQ-H\&N35) [18]. The questionnaire was originally developed by the European Organization for Research and Treatment of Cancer (EORTC) [19]. In the present study, eight of the questions, which assessed symptoms that can occur after intubation, were used. Permission to use the adjusted version was obtained from EORTC. The selected questions were the following (the original question number in the EORTC QLQ H\&N35 within parenthesis):

- Have you had pain in your mouth? (31)

- Have you had pain in your jaw? (32) 
- Have you had soreness in your mouth? (33)

- Have you had a painful throat? (34)

- Have you had problems swallowing liquids? (35)

- Have you had a dry mouth? (41)

- Have you coughed? (45)

- Have you been hoarse? (46)

Each patient indicated the extent to which he/she had experienced these symptoms or problems as "Not at all", "A little", "Quite a bit" or "Very much". The questionnaire was completed by the patients at 2 time points in time; prior to surgery (Visit 3, to cover the past $24 \mathrm{~h}$ ) and in the morning of the first post-operative day (Visit 4, to cover the period post-surgery). The baseline was established before surgery in order to be able to distinguish between the intensity of symptoms in relation to the tube alone and not the patient's condition prior to surgery.

In order to detect any changes in the tracheal mucosa correlated with the ETT, visual inspections were carried out by an anaesthesiologist/surgeon both before intubation and after extubation using a flexible fibre-optic bronchoscopy. The assessments were formulated using the following 4-graded scale:

I. No signs of irritation/inflammation of the mucosa in the trachea

II. Weak signs of irritation/inflammation of the mucosa in the trachea

III.Clear signs of irritation/inflammation of the mucosa in the trachea

IV.Extensive signs of irritation or extreme discoloration/ inflammation of the mucosa in the trachea

\section{Assessments of performance and safety}

Safety and Performance were assessed after surgery (Visit 3 and Visit 4) by noting Adverse Events, occurrence of complications during the post-operative course and any ETT handling problems experienced by the staff. It was the duty and right of the investigator to interrupt the patient's participation if the patient's health or well-being was threatened by continuation in the study. A patient could be discontinued if e.g., wrong ETT was placed by mistake. Further, concomitant medications could require a patient's discontinuation. However, each case of withdrawal was to be discussed individually with the Sponsor prior to any decision of discontinuation, unless the decision was based on safety reasons. The pre-defined reasons for patient withdrawal were as follows:

1. Serious life threatening AE, related or unrelated to the treatment in test (i.e., the ETT). This could be events such as critical bleeding, myocardial infarct, urticaria, angioedema chock and asthma bronchiale.

2. Patient's own request

3. Investigator's opinion that it is in the patient's best interest to discontinue. The reason, time and date of discontinuation had to be recorded in the eCRF.

\section{Exploratory assessments of microbial adhesion to endotracheal tubes}

Immediately after extubation, the distal piece of the ETT was cut with a sterile pair of scissors precisely above the cuff into a sterile tube containing PBS (Phosphate Buffered Saline) covering the tube to keep microorganisms alive. The container was immediately transported to the Department of Clinical Microbiology, to assess the quantity and type of pathogen and/or nonpathogen microbes. The antibiotic resistance of pathogen microbes (if those were found) was also to be determined.

At arrival in the lab, the tube was vortexed vigorously in order to remove mucus from the ETT piece. The ETT piece was then moved to a new tube containing $30 \mathrm{~mL}$ of PBS, washed by vortexing and if needed repeatedly washed until all visual mucus was removed. Thereafter it was put in another tube containing $10 \mathrm{~mL}$ PBS and $0.05 \%$ Tween-20. In order to remove the adhered microorganisms from the surface it was vortexed for $1 \mathrm{~min}$ at maximum speed. The PBS-Tween solution with the removed microorganisms was diluted and plated out according to standard procedures for clinical airway sampling at the Dept. of Clinical Microbiology, and evaluated by viable count and microbiological typing the following day [20-22].

\section{Statistical analysis}

In this exploratory study, 30 patients (20 patients in the BIP ETT group and 10 in the uncoated, standard ETT group) were judged to be needed in order to reveal statistically significant differences in the primary analyses. With an estimated drop-out rate of $20 \%$, the expected number of evaluable patients was 24. At the end, 29 patients were evaluated (19 in the BIP ETT group and 10 in the standard ETT group).

For the 8 questions used from the EORTC QLQ $H \& N 35$, the difference between the treatment groups on the day after surgery were tested with Fisher's Exact Test. Confidence intervals (95\%) of the proportion of patients for each treatment were calculated using the Clopper-Pearson method. The questions after surgery were analysed with a Proportional Odds Model, specifying a multinomial distribution and cumulative logit link. The model included the answer of the respective questions before surgery as a covariate, and tested the difference between the treatments using the likelihood ratio statistic. The proportional odds assumption was tested 
with the Score test. If more than 4 of the 8 score tests rejected the null hypothesis of proportional odds, then an extended Mantel-Haenzel analysis controlling for the EORTC QLQ H\&N35 answer before surgery were to be applied for each of the questions.

Differences between the treatment groups of assessed changes in the tracheal mucosa, on the 4-graded scale, were analysed using Fisher's Exact Test. Confidence intervals (95\%) of the proportion of patients for each treatment were calculated using the Clopper-Pearson method.

Direct assessment after extubation was also to be analysed using a Proportional Odds Model, including the evaluation/judgement prior to intubation as a covariate. However, since all patients in the standard ETT treatment group were in the same category at extubation, the Proportional Odds Model analysis was not applicable.

Adverse events (AEs) and adverse device related events (ADEs) were coded using MedDRA version 15.1. No statistical tests were carried out but an overall summary of the number and proportion of patients and the number of events in each AE category (AE, SAE (Serious AE), ADE, SADE (Serious ADE) was made. However, since only one AE was reported, no tabulation of AEs/ ADEs was carried out. Problems during the postoperative course are presented with descriptive statistics by treatment group (number and proportion of patients). Problems related to the ETT handling were presented in descriptive statistics by treatment group as the number and proportion of patients in each category (Yes/No).

\section{Results}

All patients, who had completed the study $(n=29)$, were included in the analysis set; $n=19$ in BIP ETT treatment group and $n=10$ in the standard ETT treatment group.

\section{Demographics}

The demographics, weight and height, are given in Table 1.
No deviations were found for any of the patients in either treatment group regarding physical examination or vital signs prior to surgery. In total, 4 patients in the study had concurrent diseases or relevant medical history, see Table 2 .

\section{Tolerability evaluation Modified EORTC QLQ H\&N35}

Before surgery, none of the patients from either study group reported any pain in their mouth, jaw or throat, nor did anyone report any swallowing problems. One patient in the BIP ETT group reported soreness in the mouth, $5 \%$. With regards to cough one case in the BIP ETT-group (5\%) and in two cases in the control ETT group $(20 \%)$ were reported a substantial number of patients experienced dry mouth before surgery i.e., $63 \%$ in BIP ETT group and $50 \%$ in the standard ETT group. Thus, both groups reported similar symptoms from the throat area before the surgery.

The difference between responses from the BIP ETT group and standard ETT group on the day after surgery was tested using Fisher's Exact Test. The outcome after surgery is reported in the Table 3.

A statistically significant difference was found for dry mouth ( $p$-value: 0.0434 ), suggesting more problems with dry mouth in the BIP ETT treatment group. As mentioned, a large proportion (63\%) of these patients reported symptoms before surgery. No statistically significant difference was found for the other symptoms/ problems. Fisher's Exact Test was not carried out for pain in the jaw and soreness in mouth since all patients were in the same category.

An analysis with Proportional Odds Models controlled for value/category prior to surgery for cough and painful throat symptoms. But due to non-convergence, not for the value prior to surgery concerning pain in the jaw and dry mouth. There was a statistically significant difference between the treatment groups for cough $p=$ 0.022 and for dry mouth symptoms $p=0.014$, suggesting

Table 1 Demographics and baseline characteristics

\begin{tabular}{|c|c|c|c|c|c|}
\hline & & Coated ETT & Standard ETT & Total & $p$-value \\
\hline & & $n=19$ & $n=10$ & $n=29$ & \\
\hline Age & Mean (SD) & $67.3(7.3)$ & $60.8(13.7)$ & $65.1(10.2)$ & 0.19 \\
\hline \multirow[t]{2}{*}{ Sex } & Female & $13(68 \%)$ & $5(50 \%)$ & 18 (62 \%) & 0.43 \\
\hline & Male & $6(32 \%)$ & $5(50 \%)$ & 11 (38 \%) & \\
\hline Weight (kg) & Mean (SD) & $72.1(16.2)$ & $72.0(9.7)$ & $72.0(14.1)$ & 0.98 \\
\hline Height (cm) & Mean (SD) & 166.7 (8.5) & 172.6 (8.5) & $168.7(8.8)$ & 0.09 \\
\hline \multirow[t]{3}{*}{ Smoking Status } & Never smoked & $13(68 \%)$ & $6(60 \%)$ & 19 (66 \%) & 0.84 \\
\hline & Stopped & 4 (21 \%) & 3 (30 \%) & 7 (24 \%) & \\
\hline & Yes, currently & 2 (10 \%) & 1 (10 \%) & $3(10 \%)$ & \\
\hline
\end{tabular}


Table 2 Medical/surgical history and concurrent diseases

\begin{tabular}{|c|c|c|c|c|}
\hline & Coated ETT & Standard ETT & Total & $p$-value \\
\hline & $n=19$ & $n=10$ & $n=29$ & \\
\hline System Organ Class/Preferred Term & n (\%) & n (\%) & n (\%) & \\
\hline Immune system disorders & $2(10)$ & $1(10)$ & $3(10)$ & 1 \\
\hline Allergy to metals & $1(5)$ & $1(10)$ & $2(7)$ & \\
\hline Drug hypersensitivity & $1(5)$ & 0 & $1(3)$ & \\
\hline Seasonal allergy & 0 & $1(10)$ & $1(3)$ & \\
\hline Respiratory, thoracic and mediastinal disorders & $1(5)$ & 0 & $1(3)$ & 1 \\
\hline Cough & $1(5)$ & 0 & $1(3)$ & \\
\hline Surgical and medical procedures & 0 & $1(10)$ & $1(3)$ & \\
\hline Coronary artery bypass & 0 & $1(10)$ & $1(3)$ & 0.34 \\
\hline
\end{tabular}

$n=$ Number of patients reporting the term

somewhat more problems with these aspects in the BIP ETT treatment group compared to the standard ETT group, see Table 4. Since more patients in the BIP ETT group had symptoms prior to surgery, regarding the dry mouth symptom this difference should be treated with caution.

There were no signs of irritation or inflammation of the upper airway surface tracheal lining at intubation or extubation, in the standard ETT group. In the BIP ETT group, weak signs of irritation/inflammation were seen at intubation for one patient (5.6\%). At the time of extubation, 4 patients had weak signs of irritation/inflammation. In one of the cases, however, - the evaluation prior to surgery was missing and in a second case, the patient had showed irritation already prior to surgery. There was no statistically significant difference between the BIP ETT and standard ETT treatment group ( $p$-value: 0.268 ), see Table 5.

\section{Safety and performance evaluation}

One AE was reported for one patient in the BIP ETT group during the course of the study.

A shallow wound was seen in the trachea prior to extubation, probably caused either by the mechanical impact of the fiberscope and/or the BIP ETT according to the anaesthesiologist. It was not considered a result of a difficult airway hence the patient was not excluded from the analysis set. No signs of inflammation were noted. The start date of the event was reported as the day of surgery (Visit 3), and the event was on-going at the time of the patient's study completion. The event was considered mild in severity and non-serious. No medication or treatment was given due to the $\mathrm{AE}$.

ETT handling problems were reported for 1 patient (5\%) in the BIP ETT group: there were some difficulties when pulling the ETT over the fibreoptic bronchoscope. This was however not considered serious.

\section{Exploratory assessment of microbial adhesion to endotracheal tubes}

The microbiological analysis of the ETTs after extubation showed Enterococci species, Haemophilus parainfluenzae, Neisseria species, Staphylococci and Streptococci species, see Table 6. These species are all recognized as normal flora in the airways, i.e., no pathogenic species were found on either type of ETT. Therefore, no antibiotic resistance testing was carried out. The level of bacterial colonization on the ETT surfaces was similar for both ETT types, approximately $10^{1}$ to $10^{3} \mathrm{CFU} / \mathrm{cm}^{2}$, see Figs. 1 and 2. Due to a large inter-patient variation for both tubes, a reduction of bacterial adhesion of the same magnitude as the one observed invitro was not possible to detect. As the Alpha-haemolytic Streptococci and Neisseria species were the most commonly isolated bacteria from the tubes, they were plotted/visualized in graphs, see Figs. 1 and 2.

\section{Discussion}

This was a single-centre, randomised, single-blinded, and controlled, first- in- man, post Conformity Europermit (EC) certification and CE marking study. The study was planned to address a clinical concern that the tracheal mucosa; known to be one of the bodies most sensitive and reactive tissue; might be irritated by the new coating. The tracheal surface is known to react with coughing, swelling, and irritations to many substances even in very tiny doses. So therefore, the primary endpoint in this study was; tolerability of the coated tube in the human trachea.

The major finding in this comparative clinical devicetesting study of coated BIP ETTs and uncoated ETTs, was that practically no differences in tolerability and performance could be detected during short term use, i.e., intubation 3 to $8 \mathrm{~h}$, in patients scheduled for elective abdominal surgery during general anaesthesia. This observational first-in-man study had a randomised, singleblind and controlled design, where standard, uncoated 
Table 3 Modified EORTC QLQ H\&N35. Differences between treatment groups after surgery using the Fisher's Exact Test

\begin{tabular}{|c|c|c|c|c|c|c|}
\hline \multirow[b]{3}{*}{ Parameter } & \multirow[b]{3}{*}{ Analysis value } & \multicolumn{2}{|c|}{ Coated ETT } & \multicolumn{2}{|c|}{ Standard ETT } & \multirow[b]{3}{*}{$p$-value } \\
\hline & & \multicolumn{2}{|l|}{$n=19$} & \multicolumn{2}{|l|}{$n=10$} & \\
\hline & & $\mathrm{n}(\%)$ & $95 \% \mathrm{Cl}^{\mathrm{a}}$ & $\overline{n(\%)}$ & $95 \% \mathrm{Cl}^{\mathrm{a}}$ & \\
\hline \multirow[t]{4}{*}{ Cough } & Not at all & $9(47)$ & $(24 ; 71)$ & $7(70)$ & $(35 ; 93)$ & 0.6294 \\
\hline & A little & $9(47)$ & $(24 ; 71)$ & $3(30)$ & $(7 ; 65)$ & \\
\hline & Quite a bit & 0 & $(0 ; 18)$ & 0 & $(0 ; 31)$ & \\
\hline & Very much & $1(5)$ & $(0 ; 26)$ & 0 & $(0 ; 31)$ & \\
\hline \multirow[t]{4}{*}{ Dry mouth } & Not at all & 0 & $(0 ; 18)$ & $1(10)$ & $(0 ; 44)$ & 0.043 \\
\hline & A little & 0 & $(0 ; 18)$ & $2(20)$ & $(2 ; 56)$ & \\
\hline & Quite a bit & $6(32)$ & $(13 ; 57)$ & $4(40)$ & $(12 ; 74)$ & \\
\hline & Very much & $13(69)$ & $(43 ; 88)$ & $3(30)$ & $(7 ; 65)$ & \\
\hline \multirow[t]{4}{*}{ Hoarse } & Not at all & $5(26)$ & $(9 ; 51)$ & $2(20)$ & $(2 ; 56)$ & 0.883 \\
\hline & A little & $9(47)$ & $(24 ; 71)$ & $4(40)$ & $(12 ; 74)$ & \\
\hline & Quite a bit & $3(16)$ & $(3 ; 40)$ & $3(30)$ & $(7 ; 65)$ & \\
\hline & Very much & $2(10)$ & $(1 ; 33)$ & $1(10)$ & $(0 ; 44)$ & \\
\hline \multirow[t]{4}{*}{ Pain in jaw } & Not at all & $19(100)$ & $(82 ; 100)$ & $10(100)$ & $(69 ; 100)$ & \\
\hline & A little & 0 & $(0 ; 18)$ & 0 & $(0 ; 31)$ & \\
\hline & Quite a bit & 0 & $(0 ; 18)$ & 0 & $(0 ; 31)$ & \\
\hline & Very much & 0 & $(0 ; 18)$ & 0 & $(0 ; 31)$ & \\
\hline \multirow[t]{4}{*}{ Pain in mouth } & Not at all & $19(100)$ & $(82 ; 100)$ & $9(90)$ & $(56 ; 100)$ & 0.345 \\
\hline & A little & 0 & $(0 ; 18)$ & $1(10)$ & $(0 ; 44)$ & \\
\hline & Quite a bit & 0 & $(0 ; 18)$ & 0 & $(0 ; 31)$ & \\
\hline & Very much & 0 & $(0 ; 18)$ & 0 & $(0 ; 31)$ & \\
\hline \multirow[t]{4}{*}{ Painful throat } & Not at all & $13(68)$ & $(43 ; 88)$ & $9(90)$ & $(56 ; 00)$ & 0.590 \\
\hline & A little & $5(26)$ & $(9 ; 51)$ & $1(10)$ & $(0.3 ; 44)$ & \\
\hline & Quite a bit & $1(5)$ & $(0.1 ; 26)$ & 0 & $(0 ; 31)$ & \\
\hline & Very much & 0 & $(0 ; 18)$ & 0 & $(0 ; 31)$ & \\
\hline \multirow[t]{4}{*}{ Problems swallowing liquids } & Not at all & $19(100)$ & $(82 ; 100)$ & $8(89)$ & $(52 ; 100)$ & 0.333 \\
\hline & A little & 0 & $(0 ; 19)$ & 0 & $(0 ; 34)$ & \\
\hline & Quite a bit & 0 & $(0 ; 19)$ & 0 & $(0 ; 34)$ & \\
\hline & Very much & 0 & $(0 ; 19)$ & $1(11)$ & $(0.3 ; 48)$ & \\
\hline \multirow[t]{4}{*}{ Soreness in mouth } & Not at all & $19(100)$ & $(82 ; 100)$ & $10(100)$ & $(69 ; 100)$ & \\
\hline & A little & 0 & $(0 ; 18)$ & 0 & $(0 ; 31)$ & \\
\hline & Quite a bit & 0 & $(0 ; 18)$ & 0 & $(0 ; 31)$ & \\
\hline & Very much & 0 & $(0 ; 18)$ & 0 & $(0 ; 31)$ & \\
\hline
\end{tabular}

${ }^{a}$ Confidence Interval ${ }^{b}$ Fisher's Exact Test

Fisher's Exact Test was not performed for pain in jaw and soreness in mouth since all patients were in one category

ETTs served as comparator. The primary objective was to determine the tolerability of the BIP ETT compared to the standard ETT. Symptoms from mouth, throat and airway were assessed by questionnaires and the tracheal mucosa was inspected post exposure. Furthermore, performance and a few safety aspects related to BIP ETT were assessed based on reported AEs and adverse device-related events, as well as problems during the post-operative course, and ETT handling problems experienced by the health care personnel.

\section{Tolerability and adverse events}

The modified EORTC QLQ H\&N35 is comprised of 8 questions pertaining to symptoms/problems from the ear, nose and throat areas. The questionnaire EORTC QLQ H\&N35 was primarily developed to investigate health-related quality of life issues in patients with head and neck cancer and is very well validated and used in many studies $[23,24]$. We chose eight questions as they covered symptoms that may occur after an intubation. The frequency of symptoms was as expected an increase 
Table 4 Modified EORTC QLQ H\&N35 Difference between treatments day after surgery, Proportional Odds Model

\begin{tabular}{lclc}
\hline Parameter & Odds Ratio $^{\mathrm{a}}$ & $95 \% \mathrm{Cl}^{\mathrm{b}}$ & $p$-value \\
\hline Cough & 0.082 & $0.003,0.726$ & 0.022 \\
Dry mouth & 0.14 & $0.02,0.67$ & 0.014 \\
Hoarse & 1.56 & $0.38,6.62$ & 0.535 \\
Painful throat & 0.24 & $0.01,1.71$ & 0.165 \\
\hline
\end{tabular}

${ }^{\mathrm{a}}$ Odds ratio between coated ETT and standard ETT (ratio of the odds of being in the lower categories). The model controls for baseline for the parameters Cough and Painful throat but due to non-convergence, baseline was not included in the Dry mouth and Hoarseness models. The Proportional Odds Model was only applied to the symptoms/problems when not all patients within a treatment group were in the same category

${ }^{b}$ Likelihood ratio based confidence interval

in both groups, and some differences in cough and dry mouth was observed. The difference should be treated with caution because of small study groups in combination with a statistical model, which could not correct the differences in baseline (before surgery) due to nonconvergence. No known attributes of the BIP ETT have been identified that rationally could explain the possibly increased problems with cough and dry mouth. However, the design of the two ETTs differed slightly, which could potentially have played a role.

Further, there was no statistically significant difference regarding the appearance of the tracheal mucosa between the 2 treatment groups at extubation. Safety was also evaluated by AE and ADE incidence and the occurrence of any post-operative. No severe AEs were noted post-operatively in either of the treatment groups.

\section{Microbial assessment}

In order to assess microbial adhesion to the ETTs a distal piece of the ETT was cut off after extubation. The quantity and type of pathogen and non-pathogen bacteria were assessed. The microbiological testing found Enterococci, Haemophilus parainfluenzae, Neisseria, Staphylococci, and
Streptococci species, which are species all recognized as normal flora in the airways [25-27]. No pathogenic species were found therefore no antibiotic resistance testing was carried out.

The level of bacterial colonization on the ETT surfaces was similar for both types of tubes, approximately $10^{1}$ to $10^{3} \mathrm{CFU} / \mathrm{cm}^{2}$ and overall low compared to other studies where the tracheal colonization has been investigated [25-27]. We found a large inter-patient variation with regards to microbial adhesion for both types of surfaces and it is known that the normal flora differs between patients with regards to both the species found and the amount of each species. In vitro experiments have shown significant reduction in adhesion of several species, both Gram positive and Gram negative to the coated BIP ETT, (unpublished data).

The time of intubation in the current study was between 3 and $8 \mathrm{~h}$, (mean $5 \mathrm{~h}$ and $14 \mathrm{~min}$ ) which is short when evaluating microbial adherence in vivo. Bacterial biofilms have been shown to develop on ETTs after around $24 \mathrm{~h}$, and it is this advanced form of microbial adhesion and subsequent detachment that play a part in the development of infection [28]. Further, the definition of VAP (Ventilator Associated Pneumonia) requires a time frame of at least 24 of intubation [29]. In conclusion, the used microbial assessments were found easy to employ, and detected reasonable bacterial colonisation even after short periods of intubation.

\section{Clinical relevance/importance, study limitations and future studies}

The primary purpose of this study was to establish whether the noble metal alloy coated BIP ETT could be well tolerated during short-term (up to $8 \mathrm{~h}$ ) clinical use in anaesthesia and elective surgery [30, 31]. This was considered necessary before further trials of the device could be carried out in a potentially more complex study

Table 5 Upper airway unblinded judgement, Fisher's Exact Test

\begin{tabular}{|c|c|c|c|c|c|c|}
\hline \multirow[b]{3}{*}{ Parameter } & \multirow[b]{3}{*}{ Analysis value } & \multicolumn{2}{|c|}{ Coated ETT } & \multicolumn{2}{|c|}{ Standard ETT } & \multirow[b]{3}{*}{$p$-value } \\
\hline & & \multicolumn{2}{|l|}{$n=19$} & \multicolumn{2}{|l|}{$n=10$} & \\
\hline & & $\overline{n(\%)}$ & $95 \% \mathrm{Cl}^{\mathrm{a}}$ & $\overline{n(\%)}$ & $95 \% \mathrm{Cl}^{\mathrm{a}}$ & \\
\hline \multirow[t]{4}{*}{ Intubation } & 1 & $17(94)$ & $(73,100)$ & $10(100)$ & $(69 \% 100)$ & \\
\hline & $\|$ & $1(6)$ & $(0,27)$ & 0 & $(0,31)$ & . \\
\hline & III & 0 & $(0,19)$ & 0 & $(0,31)$ & \\
\hline & IV & 0 & $(0,19)$ & 0 & $(0,31)$ & \\
\hline \multirow[t]{4}{*}{ Extubation } & 1 & $15(79)$ & $(54,94)$ & $10(100)$ & $(69,100)$ & . \\
\hline & $\|$ & $4(21)$ & $(6,46)$ & 0 & $(0,31)$ & . \\
\hline & III & 0 & $(0,18)$ & 0 & $(0,31)$ & . \\
\hline & IV & 0 & $(0 \%, 18 \%)$ & 0 & $(0 \%, 31 \%)$ & 0.2680 \\
\hline
\end{tabular}

${ }^{a}$ Confidence intervals calculated using Clopper-Pearson method. ${ }^{\mathrm{b}}$ Fisher's Exact Test. Only provided for extubation

I - No signs of irritation/inflammation of the mucosa in the trachea; II - Weak signs of irritation/inflammation of the mucosa in the trachea; III - Clear signs of irritation/inflammation of the mucosa in the trachea; IV - Extensive signs of irritation or extreme discoloration/inflammation of the mucosa in the trachea 
Table 6 Microbiological isolation from endotracheal tubes

\begin{tabular}{lll}
\hline Microorganism & $\begin{array}{l}\text { Coated ETT, } \\
n=19(\%)\end{array}$ & $\begin{array}{l}\text { Standard ETT, } \\
n=10(\%)\end{array}$ \\
\hline Alpha-hemolytic Streptococcus & $18(95)$ & $10(100)$ \\
Neisseria species & $11(58)$ & $8(80)$ \\
Enterococci species & $1(5)$ & $0(0)$ \\
Haemophilus parainfluenzae & $2(11)$ & $0(0)$ \\
Coagulase-negative Staphylococci & $4(16)$ & $1(10)$ \\
\hline
\end{tabular}

group consisting of ICU patients, with longer intubation periods. Knowing the degree of sensitivity in the trachea a patient centred outcome seemed reasonable. As the human trachea had not been exposed to the coating before there was no observation that could be used as a base for a proper power analysis. We considered an observational study on at least 16 patients with 8 controls to be reasonable. As we were eager not to have fewer observations than that, we screened and included 30 patients expecting a drop-out rate of $20 \%$. However we ended up with a drop-out rate of only $3 \%$. To add an explanatory measure to eventual patient-experienced symptoms we performed a pre- and post-intubation bronchoscopy.

A methodological limitation in the study is that we intubated the patients after bronchoscopy, using the previously inserted bronchoscope to guide the tube into the trachea. This could lead to contamination of the tube and the trachea with bacteria from the mouth. Despite the possibility of contamination by the bronchoscope, the identified bacteria do not appear of clinical relevance. We do not think it has affected our results as the study was randomized, there was a control group and the same methodology was used in both groups. Other limitations of the current study include the small sample size, uneven treatment group sizes and the short intubation time and unblended inspection of the tracheal mucosa. Hence, the next step is to carry out a study in a larger population, with a longer intubation period $(>24 \mathrm{~h}$ ) and in a patient group during ICU care to enable evaluation of a possible reduction in VAP.

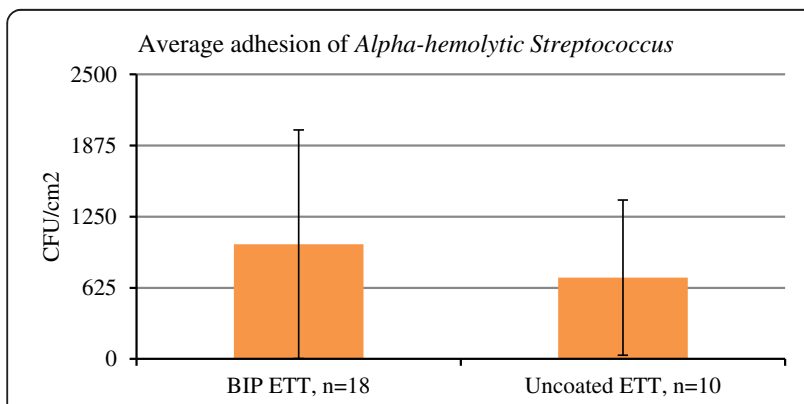

Fig. 1 In vivo colonization of Streptococcus presented as CFU/cm². Standard deviation is displayed

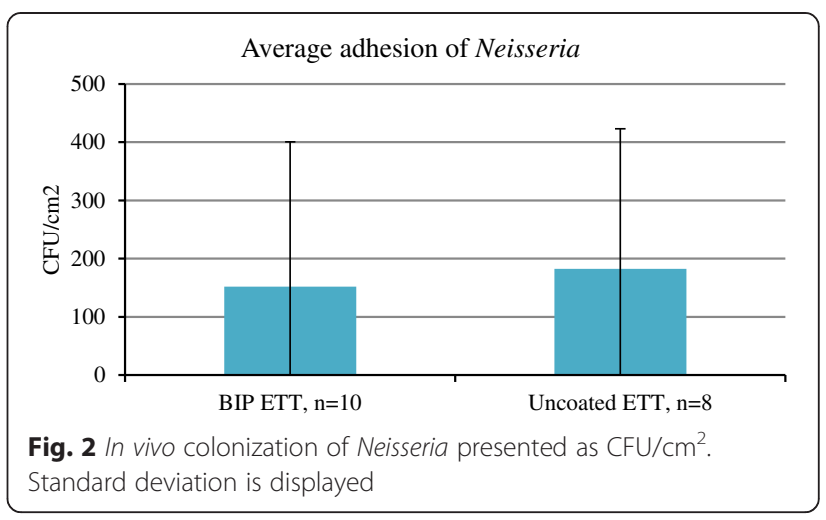

\section{Conclusions}

We compared tolerability and functional performance between a new, coated endotracheal tube (BIP ETT) and a standard endotracheal tube (ETT), and found minor differences during short-term use, 3 to $8 \mathrm{~h}$ of intubation. The differences were observed only in 2 of 8 symptoms - in coughing, $p=0.022$ and possibly in dry mouth $(p=0.014$, but with presence of statistical confounders), while there was no difference in adverse events and no mucosal damage. The results should be treated with caution due to a small study size. Further, the current study had too short intubation periods and too small sample sizes to detect differences in the reduction of bacterial adhesion and biofilm formation due to large interindividual variability in the results. In summary, the study shows that the BIP ETT is well tolerated and has good performance. Studies of larger sample sizes and longer intubation periods $(>24 \mathrm{~h})$ in the ICU-setting can now be planned for the evaluation of the possible efficacy of the BIP ETT in reducing bacterial colonisation and prevention of VAP.

\section{Key messages}

Healthcare associated infections continue to play a major role in complicating hospital care. The surface of various devices can be modified in order to reduce bacterial colonization and subsequent infection. We compared new surface-coated endotracheal tubes to standard devices during elective surgery for 3-8 h, and found insignificant differences in tolerability and performance. Only larger studies with longer exposure times in special patient populations (ICU) could demonstrate possible benefits of the surface coating in reduction of VAP and produce experience supporting extrapolation of conclusion on safety from this limited study into general patient population.

\section{Abbreviations}

AE: Adverse Events; BIP: Bactiguard Infection Protection; CE: Conformité Européenne; CFU: Colony Forming Units; EORTC: European Organization for Research and Treatment of Cancer; ETT: Endotracheal Tube; GCP: Good Clinical Practice; HAl: Hospital Acquired Infections; ICU: Intensive Care Units; 
NSAID: Non-Steroid Anti-Inflammatory Drugs; PBS: Phosphate Buffered Saline; PCG: Pharma Consulting Group; QLQ-H\&N35: Quality of Life Head and Neck Module; SAE: Serious Adverse Events; SADE: Serious Adverse Device related Events; VAP: Ventilator Associated Pneumonia.

\section{Competing interests}

Authors DJ and LB were employed by Bactiguard AB during the study. Remaining authors did not receive financial support or other benefits than sharing unrestricted grant support for materials (no salary) from the above mentioned company during the study. Bactiguard has outsourced activities such as study monitoring, data management, statistics and GCP report writing to an independent accredited clinical CRO, Pharma Consulting Group (PCG). Legal and financial agreements were signed before start of the study between Bactiguard and PCG, and Bactiguard and study site (Karolinska Hospital Huddinge). Post approval study commitment.

\section{Authors' contributions}

Study planning: GB, SK, LB, CF, SJ, DJ. Data collection: IK, SJ, SK, LB. Data analyses: GB, SK, CF, LB, SJ. Data interpretation: GB, SK, CF, LB, DJ. Manuscript preparation: GB, SK, LB, DJ, CF, IK, SJ. All authors read and approved the final manuscript.

\section{Acknowledgements}

Unrestricted grant from Bactiguard AB, Stockholm. We thank Linda Ekman, as well as Brenda Ekman for editing the language in the manuscript.

\section{Author details}

${ }^{1}$ The Swedish Red Cross University College, P.O. Box 55676SE-10215 Stockholm, Sweden. ${ }^{2}$ Karolinska Institutet, Department of Clinical Sciences, Division of Anaesthesia and Intensive Care, Danderyd Hospital, SE-18288 Stockholm, Sweden. ${ }^{3}$ Bactiguard AB, Stockholm, Sweden. ${ }^{4}$ P.O Box 15 SE-146 21 Tullinge, Sweden. ${ }^{5}$ Karolinska Institutet, Division of Clinical Microbiology, Department of Laboratory Medicine, SE-14186 Stockholm, Sweden. ${ }^{6}$ Department Anesthesia and Intensive Care, Karolinska University Hospital Huddinge, SE-14186 Stockholm, Sweden. ${ }^{7}$ CLINTEC, Karolinska Institutet, SE-14186 Stockholm, Sweden.

\section{Received: 17 April 2015 Accepted: 24 November 2015 \\ Published online: 01 December 2015}

\section{References}

1. Amato-Gauci A, Ammon A. ECDC to launch first report on communicable diseases epidemiology in the European Union. Euro Surveill Bull Eur Sur Mal Transm Eur Commun Dis Bull. 2007;12:E070607.2.

2. Klevens RM, Edwards JR, Richards Jr CL, Horan TC, Gaynes RP, Pollock D, et al. Estimating health care-associated infections and deaths in U.S. hospitals, 2002. Public Health Rep. 2007:122:160-6.

3. The National Board of Health and Welfare. Att förebygga vårdrelaterade infektioner: ett kunskapsunderlag. Report 2006-123-12. Published by The National Board of Health and Welfare, Stockholm. Sweden. ISBN 91-8548214-5.

4. Struwe J, Dumpis U, Gulbinovic J, Lagergren A, Bergman U. Healthcare associated infections in university hospitals in Latvia, Lithuania and Sweden: a simple protocol for quality assessment. Euro Surveill Bull Eur Sur Mal Transm Eur Commun Dis Bull. 2006;11:167-71.

5. Deem S, Treggiari MM. New endotracheal tubes designed to prevent ventilator-associated pneumonia: do they make a difference? Respir Care. 2010;55:1046-55.

6. Gil-Perotin S, Ramirez P, Marti V, et al. Implications of endotracheal tube biofilm in ventilator-associated pneumonia response: a state of concept. Crit Care Lond Engl. 2012;16:R93.

7. Costerton JW, Stewart PS, Greenberg EP. Bacterial biofilms: a common cause of persistent infections. Science. 1999;284:1318-22.

8. Lindsay D, von Holy A. Bacterial biofilms within the clinical setting: what healthcare professionals should know. J Hospital Infect. 2006;64:313-25.

9. Pittet $\mathrm{D}$ et al. Considerations for a WHO European strategy on healthcare-associated infection, surveillance, and control. Lancet Infect Dis. 2005;5:242-50.

10. Pittet D. Infection control and quality health care in the new millennium. Am J Infect Control. 2005:33:258-67.
11. Kollef MH, Afessa B, Anzueto A, et al. Silver-coated endotracheal tubes and incidence of ventilator-associated pneumonia: the NASCENT randomized trial. J Am Med Assoc. 2008;300:805-13.

12. Rello J, Kollef M, Diaz E, et al. Reduced burden of bacterial airway colonization with a novel silver-coated endotracheal tube in a randomized multiple-center feasibility study. Crit Care Med. 2006;34:2766-72.

13. Berra $L$, Kolobow $T$, Laquerriere $P$, et al. Internally coated endotracheal tubes with silver sulfadiazine in polyurethane to prevent bacterial colonization: a clinical trial. Intensive Care Med. 2008;34:1030-37.

14. Stenzelius K et al. Noble metal alloy-coated latex versus silicone Foley catheter in short-term catheterization: a randomized controlled study. Scand J Urol Nephrol. 2011;45(4):258-64.

15. Karchmer TB, Giannetta ET, Muto CA, Strain BA, Farr BM. A randomized crossover study of silver-coated urinary catheters in hospitalized patients. Arch Intern Med. 2000;160:3294-98.

16. Schumm K, Lam TBL. Types of urethral catheters for management of shortterm voiding problems in hospitalized adults: a short version Cochrane review. Neurourol Urodyn. 2008;27:738-46.

17. Goldschmidt $H$, Hahn U, Salwender HJ, et al. Prevention of catheter-related infections by silver coated central venous catheters in oncological patients. Zentralblatt Für Bakteriol Int J Med Microbiol. 1995;283:215-23.

18. Aaronson NK, Ahmedzai B, Bergman B, et al. The European Organization for Research and Treatment of Cancer QLQ-C30: a quality of life instrument for use in international clinical trials in oncology. J Natl Cancer Inst. 1993;85: 365-76.

19. Bjordal K, Kaasa S. Psychometric validation of the EORTC Core Quality of Life Questionnaire, 30-item version and a diagnosis-specific module for head and neck cancer patients. Acta Oncol. 1992;31:311-21.

20. Murray P. Manual in clinical microbiology. 8th ed. Washington (DC): ASM Press; 2003. p. 2322.

21. Croxatto A, Prod'hom G, Greub G. Applications of MALDI-TOF mass spectrometry in clinical diagnostic microbiology. FEMS Microbiol Rev. 2012; 36:380-407.

22. Gabriel MM et al. Effects of silver on adherence of Bacteria to Urinary Catheters: In vitro studies. Curr Microbiol. 1995:30:17-22.

23. Sherman A, Simonton S, Camp Adams D, Vural E, Owens B, Hanna E. Assessing quality of life in patients with head and neck cancer. Arch Otolaryngol Head Neck Surg. 2000;126:459-67.

24. Singer S, Ingacio Arraras J, Chie WC, et al. Performance of the EORTC questionnaire for the assessment of quality of life in head and neck cancer patients EORTC QLQ-H\&N35: a methodological review. Qual Life Res. 2013; 22:1927-41.

25. Feldman C, Kassel M, Cantrell J, et al. The presence and sequence of endotracheal tube colonization in patients undergoing mechanical ventilation. Eur Respir Rev. 1999;13:546-51

26. Björling G, Belin AL, Hellstrom C, Schedin U, Ransjo U, Alenius M, et al. Tracheostomy inner cannula care: a randomized crossover study of two decontamination procedures. Am J Infect Control. 2007:35:600-5.

27. Wilson A, Gray D, Karakiozis J, Thomas J. Advanced endotracheal tube biofilm stage, not duration of intubation, is related to pneumonia. J Trauma. 2012;72:916-23.

28. Pneumatikos IA, Dragoumanis CK, Bouros DE. Ventilator-associated pneumonia or endotracheal tube-associated pneumonia? An approach to the pathogenesis and preventive strategies emphasizing the importance of endotracheal tube. Anesthesiology. 2009;110:673-80.

29. American Thoracic Society Infectious Diseases Society of A. Guidelines for the management of adults with hospital-acquired, ventilator-associated, and healthcare-associated pneumonia. Am J Respir Crit Care Med. 2005; 171:388-416.

30. Ansari $L$ et al. The effect of endotracheal tube cuff pressure control on postextubation throat pain in orthognathic surgeries: a randomized doubleblind controlled clinical trial. Br J Oral and Maxillofac Surg. 2014;52:140-3.

31. Liu J et al. Correlations between controlled endotracheal tube cuff pressure and post procedural complications: a multicenter study. Anesth Analg. 2010;111(5):1133-7. 